\title{
Transcranial sonography changes in patients with Wilson's Disease during de-coppering therapy
}

\author{
Marta Skowrońska, Tomasz Litwin, Iwona Kurkowska-Jastrzębska, Anna Członkowska \\ Institute of Psychiatry and Neurology, Warsaw, Poland
}

\begin{abstract}
Introduction. Wilson's Disease (WD) is an inherited disorder of impaired hepatic copper metabolism that leads to copper accumulation in organs such as the liver and brain. Using transcranial sonography (TCS), we investigated brain changes in WD patients during de-coppering treatment.

Methods. Forty-one consecutive treatment-naïve WD patients were classified as having hepatic (WDh; $n=20$ ) or neurological WD (WDn; $n=21$ ) based on symptoms at diagnosis; all patients received either D-penicillamine or zinc sulfate and were observed for 24 months. TCS was performed at regular intervals from study entry (month 0) to month 24 .

Results. At study entry, bilateral lenticular nucleus (LN) hyperechogenicity was found in 18 patients with WDn and in nine with WDh $(p=0.006)$. Substantia nigra (SN) hyperechogenicity was found in nine patients with WDn) and four with WDh ( $p=n s)$. After 24 months of treatment, bilateral LN hyperechogenicity was still present in 17 patients with WDn and 14 with WDh ( $p=n s$ ). SN hyperechogenicity was present in one patient with WDn and two with WDh $(p=n s)$. The decrease in the number of patients with SN hyperechogenicity was significant in the WDn group $(p<0.05)$.
\end{abstract}

Conclusions. LN hyperechogenicity is the most common TCS abnormality in WD patients, and was observed despite two years of de-coppering treatment. SN hyperechogenicity was less common, and decreased after treatment introduction.

Key words: transcranial sonography, Wilson's Disease, therapy, substantia nigra, lenticular nucleus

(Neurol Neurochir Pol 2020; 54 (2): 185-192)

\section{Introduction}

Wilson's Disease (WD) is an autosomal recessive inherited disorder of hepatic copper metabolism that is caused by malfunction of a putative copper-transporting P-type ATPase (ATP7B). The cellular damage is thought to be due to copper deposition in affected tissues, principally the liver and the brain $[1,2]$. Clinical manifestations of WD include neurological, hepatic and psychiatric symptoms.

WD can be successfully treated with pharmacological agents, such as chelators that induce urinary excretion of copper, or zinc salts that inhibit copper absorption in the digestive tract [3]. After treatment introduction, clinical improvement is usually observed, but in some cases worsening occurs $[4,5]$.

Brain magnetic resonance imaging (MRI) in most WD patients with the neuropsychiatric form, and some with the hepatic and presymptomatic forms, show lesions, mostly in the basal ganglia [6-8]. These changes may also improve after treatment introduction $[6,9,10]$.

Lesions in midbrain structures may also be detected by transcranial sonography (TCS). This simple and safe technique is applied for the diagnosis of a number of neurodegenerative diseases. It detects hyperechogenicity of midbrain structures, including the substantia nigra (SN), thalamus, lenticular nucleus (LN), and caudate nucleus, and can be used to determine the diameter of the third ventricle or the frontal horn of the lateral ventricle [11]. In WD patients, $\mathrm{LN}$ is affected in most cases, with bilateral hyperechogenicity. Less often, changes in $\mathrm{SN}$, thalamus and red nucleus are described [12-14].

\section{Aim}

Because de-coppering treatment can improve MRI brain lesions in WD, we examined the pattern of TCS in 
treatment-naïve patients. The aim of this study was to monitor changes after the introduction of treatment. We also wanted to determine if there was a correlation between neurological deterioration or improvement and TCS results.

\section{Methods}

\section{Patients}

The study group consisted of consecutive treatment-naïve WD patients who were all diagnosed with WD based on a combination of abnormal copper results, the presence of a Kayser-Fleischer ring, and genetic testing performed at our centre as previously described $[4,5,15]$, and then confirmed using international criteria [16]. Included WD patients were classified as having either hepatic (WDh) or neurological (WDn) manifestations according to the presence of neurological/psychiatric symptoms at diagnosis.

All patients were treated with either D-penicillamine (DPA) or zinc sulfate (ZS). There was no protocol for choosing the first-line therapy and the attending neurologist had no clear preference of one drug over the other. Hepatic involvement was classified as previously described: 0 ) lack of hepatic signs or symptoms; 1 ) mild injury (increased level of aminotransferases or bilirubin or international normalised ratio [INR], but without significant sonographic changes in liver parenchyma); 2) moderate injury (increased levels of aminotransferases or bilirubin or INR $<2.4$ combined with enlargement of liver or spleen with ultrasound); 3) severe injury (INR > 2.4, and/or ultrasound changes in liver echogenicity); and 4) very severe injury (acute liver failure, hepatic encephalopathy) [4, 5]. Neurological evaluation was based on the Unified Wilson's Disease Rating Scale (UWDRS): part II, activities of daily living; and part III, neurological deficits [17]. The assessment was done at diagnosis (i.e. treatment initiation) and again at six, 12, 18, and 24 months. Neurological worsening was defined as any deterioration in UWDRS part II score or deterioration of at least four points in UWDRS part III score $[4,5]$. At each time point, patients had copper metabolism analysis consisting of serum ceruloplasmin level, serum copper level, and 24-hour copper excretion level. This study was approved by the local ethics committee.

\section{Brain imaging}

TCS was performed through the preauricular acoustic bone window using a 2.5-MHz phased-array transducer (Vivid 7; GE, USA). The chosen ultrasound parameters were a penetration depth of $14-16 \mathrm{~cm}$ and a dynamic range of $50 \mathrm{~dB}$. Image contrast and brightness were adjusted to obtain the best image. SN echogenic size measurements were performed automatically on axial TCS scans after manually encircling the outer circumference of the SN echogenic area. For the ultrasound system used, SN echogenic areas of $\geq 0.25 \mathrm{~cm}^{2}$ were considered hyperechogenic, those $\leq 0.23 \mathrm{~cm}^{2}$ were considered normal, and intermediate-sized areas were considered moderately hyperechogenic. The globus pallidus and putamen were assessed as one structure, the LN, as in the TCS protocols. The LN and thalamus were visualised in the third ventricular plane. The echogenicity of the LN and thalamus were classified as hyperechogenic when the echogenicity was more intense than the surrounding white matter. The area of hyperechogenicity was measured by manually encircling the outer circumference of the hyperechogenic area. The width of the third ventricle was measured on the axial scanning plane; width $<0.7 \mathrm{~cm}$ was considered normal (Fig.1). TCS assessment was done at study entry (i.e. treatment initiation at month 0 ) and again at months $6,12,18$, and 24 .

Since the study design involved recording two measurements for both sides, we present the result for both sides.

\section{Statistical analysis}

Statistical analysis was performed using SAS version 13.2. A $p$ value of $<0.05$ was considered to indicate statistical significance. Wilcoxon Test and Fisher's Exact Test were used to compare WD and controls. A generalised linear model (GLM) was chosen as the basic model to compare hyperechogenicity of analysed structures. An optimal model comprising the Gaussian error and the log-link function was chosen from the GLM family based on the Akaike Information Criterion (AIC), and the deviance was examined to ascertain whether the model was sufficient.

\section{Results}

The WDn group consisted of 21 patients. One patient developed a severe neurological dystonic state and died 18 months after treatment introduction. The WDh group consisted of 20 patients. One patient died 12 months after treatment introduction due to hepatic cancer.

\section{Baseline characteristics}

Baseline characteristics are shown in Table 1. WDn patients were older compared to WDh patients. Mean disease duration, from first symptoms of the disease obtained from medical history to diagnosis, was longer for neurological patients compared to hepatic ones. Both groups had hepatic involvement. In the WDn group, mean UWDRS was 4 points for part II and 18 points for part III. There was a treatment bias, as WDn patients were treated with DPA more often than WDh patients.

\section{Imaging findings}

TCS baseline and follow up results are set out in Table 2 . At the baseline, hyperechogenicity of SN was present in nine cases in the WDn group and five cases in the WDh group. In addition, mean area of SN was higher in the WDn group compared to the WDh group. Bilateral hyperechogenicity of 

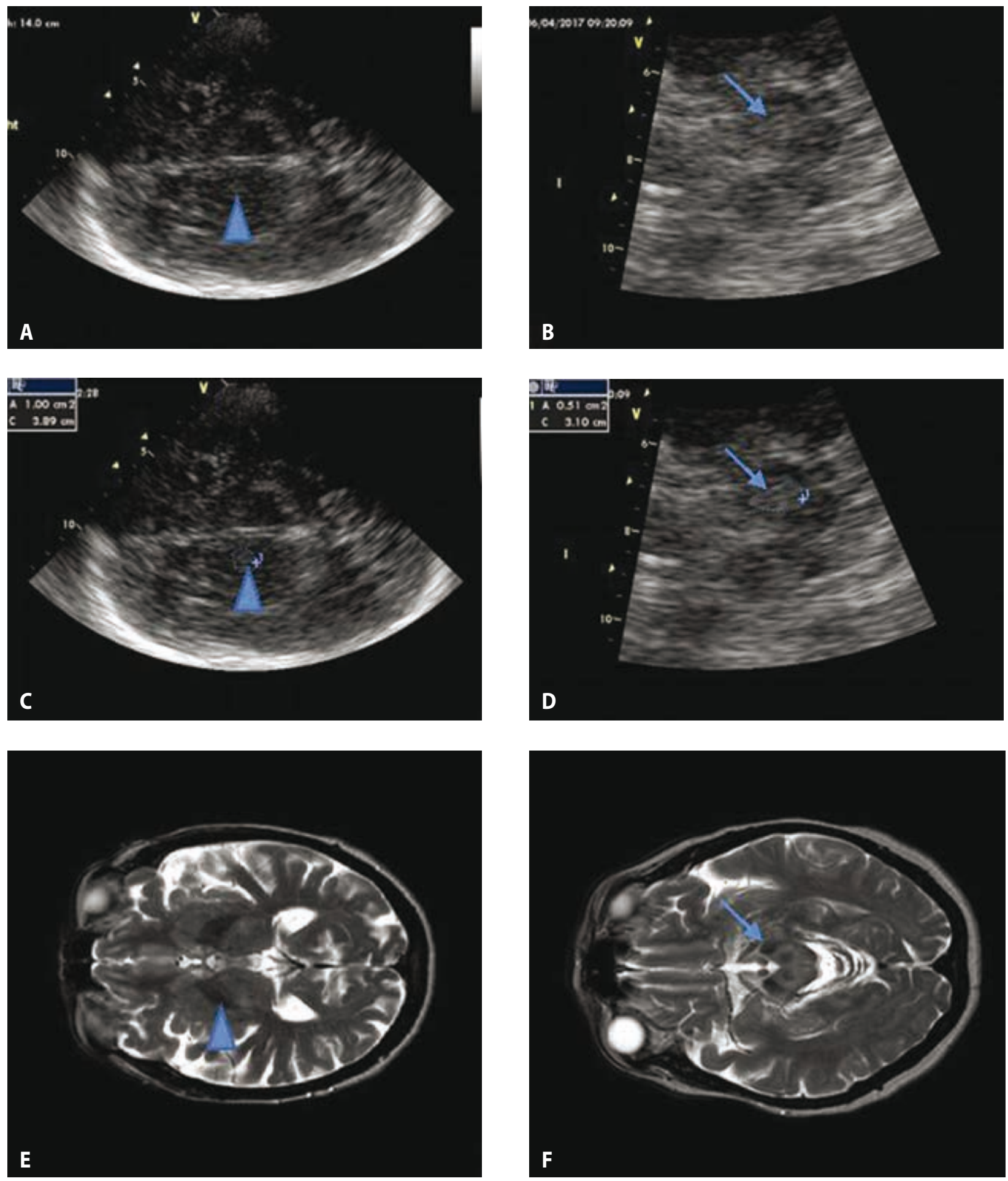

Figure 1. Transcranial sonography (TCS) images with corresponding magnetic resonance imaging (MRI) results. A and B - Representative TCS image of lenticular nucleus, where hyperechogenic part measured is shown by arrowhead; C - corresponding MRI results (T2) with hypointense signal in globus pallidus shown by arrowhead; $D$ and $E$ - Representative TCS image of substantia nigra, where hyperechogenic part measured is shown by arrowhead; F - corresponding MRI results (T2) with hypointense signal in substantia nigra shown by arrowhead 
Table 1. Baseline characteristics of study groups

\begin{tabular}{|c|c|c|c|}
\hline & $\begin{array}{l}\text { WDn } \\
(n=21)\end{array}$ & $\begin{array}{l}\text { WDh } \\
(n=20)\end{array}$ & $\begin{array}{l}\text { p value for } \\
\text { WDn vs. WDh }\end{array}$ \\
\hline Age, years, mean $\pm S D$ & $38.1 \pm 12.8$ & $32.2 \pm 11.0$ & 0.213 \\
\hline Female, n (\%) & $13(62)$ & $15(75)$ & 0.367 \\
\hline Age at onset, years, mean \pm SD & $30.9 \pm 12.1$ & $29.5 \pm 12.1$ & 0.632 \\
\hline Symptom duration before treatment, years, mean \pm SD & $5.9 \pm 8.3$ & $2.0 \pm 3.4$ & 0.036 \\
\hline Treatment with D-penicillamine, $\mathrm{n}(\%)$ & $15(71)$ & $7(35)$ & 0.019 \\
\hline Hepatic involvement, $\mathrm{n}$ & & & 0.006 \\
\hline 0 & 6 & 0 & \\
\hline 1 & 0 & 6 & \\
\hline 2 & 4 & 7 & \\
\hline 3 & 10 & 7 & \\
\hline 4 & 1 & 0 & \\
\hline UWDRS II score & $3.9 \pm 7.3$ & 0 & 0.001 \\
\hline UWDRS III score & $18.4 \pm 20.2$ & $0^{*}$ & $<0.0001$ \\
\hline
\end{tabular}

WDn - Wilson's Disease patients with neurological manifestations; WDh — Wilson's Disease patients with hepatic manifestations; SD — standard deviation; UWDRS, United Wilson's Disease Rating Scale *One patient had 2 points for postural tremor

Table 2. Transcranial sonography results

\begin{tabular}{|c|c|c|c|c|c|c|c|c|}
\hline & $\begin{array}{l}\text { WDn M0 } \\
(n=21)\end{array}$ & $\begin{array}{l}\text { WDn M24 } \\
(n=20)\end{array}$ & $\begin{array}{l}\text { WDn: } \\
\text { p value } \\
\text { M0/M24 }\end{array}$ & $\begin{array}{l}\text { WDh M0 } \\
(n=20)\end{array}$ & $\begin{array}{l}\text { WDh M24 } \\
(n=19)\end{array}$ & $\begin{array}{l}\text { WDh: } \\
\text { p value } \\
\text { M0/M24 }\end{array}$ & $\begin{array}{l}\text { p value } \\
\text { WDn/WDh } \\
\text { M0 }\end{array}$ & $\begin{array}{l}\mathrm{p} \text { value for } \\
\text { WDn/WDh } \\
\text { M24 }\end{array}$ \\
\hline \multicolumn{9}{|l|}{ Substantia nigra } \\
\hline Right, $n$ & & & & & & & 0.273 & 0.531 \\
\hline$<0.23 \mathrm{~cm}^{2}$ & 11 & 17 & 0.024 & 14 & 14 & 1.00 & & \\
\hline $0.23-0.25 \mathrm{~cm}^{2}$ & 1 & 2 & 0.051 & 2 & 3 & 0.292 & & \\
\hline$>0.25 \mathrm{~cm}^{2}$ & 9 & 1 & 0.005 & 4 & 2 & 0.671 & & \\
\hline Area, $\mathrm{cm}^{2}$, mean $\pm \mathrm{SD}$ & $0.23 \pm 0.06$ & $0.19 \pm 0.04$ & 0.0434 & $0.20 \pm 0.05$ & $0.20 \pm 0.05$ & 0.759 & 0.199 & 0.644 \\
\hline Left (n) & & & & & & & 0.049 & 0.068 \\
\hline$<0.23 \mathrm{~cm}^{2}$ & 8 & 19 & $<0.001$ & 14 & 14 & 1.00 & & \\
\hline $0.23-0.25 \mathrm{~cm}^{2}$ & 7 & 0 & ns & 1 & 3 & 0.267 & & \\
\hline$>0.25 \mathrm{~cm}^{2}$ & 6 & 1 & 0.045 & 5 & 2 & 0.239 & & \\
\hline Area, $\mathrm{cm}^{2}$, mean $\pm \mathrm{SD}$ & $0.23 \pm 0.06$ & $0.17 \pm 0.04$ & $<0.0001$ & $0.21 \pm 0.05$ & $0.21 \pm 0.04$ & 0.685 & 0.273 & 0.011 \\
\hline \multicolumn{9}{|l|}{ Lenticular nucleus } \\
\hline Bilateral changes, $n$ & 18 & 17 & 0.948 & 9 & 14 & 0.783 & 0.006 & 0.381 \\
\hline Right area, $\mathrm{cm}^{2}$, mean $\pm \mathrm{SD}$ & $0.60 \pm 0.33$ & $0.63 \pm 0.34$ & 1.0 & $0.45 \pm 0.42$ & $0.68 \pm 0.40$ & 0.089 & 0.229 & 0.461 \\
\hline Left area, $\mathrm{cm}^{2}$, mean $\pm \mathrm{SD}$ & $0.74 \pm 0.45$ & $0.55 \pm 0.28$ & 0.242 & $0.31 \pm 0.35$ & $0.47 \pm 0.35$ & 0.107 & 0.005 & 0.635 \\
\hline \multicolumn{9}{|l|}{ Thalamus } \\
\hline Bilateral changes, $n$ & 6 & 3 & 0.294 & 1 & 1 & 1.00 & 0.001 & 0.316 \\
\hline Right area, $\mathrm{cm}^{2}$, mean $\pm \mathrm{SD}$ & $0.20 \pm 0.25$ & $0.08 \pm 0.17$ & 0.053 & $0.06 \pm 0.15$ & $0.05 \pm 0.14$ & 1.00 & 0.034 & 0.438 \\
\hline Left area, $\mathrm{cm}^{2}$, mean $\pm \mathrm{SD}$ & $0.19 \pm 0.30$ & $0.15 \pm 0.27$ & 0.641 & $0.06 \pm 0.15$ & $0.11 \pm 0.23$ & 0.687 & 0.115 & 0.561 \\
\hline
\end{tabular}

WDn - Wilson's Disease patients with neurological manifestations; WDh — Wilson's Disease patients with hepatic manifestations; SD — standard deviation; ns — not significant; M0 — month 0; M24 — month 24

LN was found in 18 WDn patients and nine WDh patients (an additional six WDh patients had unilateral hyperechogenicity) and thalamic hyperechogenicity was found in six WDn patients and this was significant compared to WDh (one patient).

The only correlation between TCS hyperechogenicity and copper metabolism was found to be between SN hyperechogenicity and 24-h copper excretion results. This was in patients with WDn $\left(\mathrm{R}^{2}=0.1473, \mathrm{p}=0.086\right.$ for SN left and $\mathrm{R}^{2}=0.2843, \mathrm{p}=0.013$ for $\mathrm{SN}$ right) and patients with WDh $\left(R^{2}=0.3928, p=0.003\right.$ for SN left and $R^{2}=0.2265$, $\mathrm{p}=0.034$ for $\mathrm{SN}$ right). 
After 24 months of follow-up, none of the WDh patients had developed neurological symptoms. In the WDh group, three patients had deteriorated, three had stabilised, and 14 had improved. In the WDn group, four patients had deteriorated, five had stabilised, and 12 had improved in terms of UWDRS part III score.

At 24 months, the number of patients with SN hyperechogenicity had decreased. Also, mean area of SN decreased over time in the WDn group but not in the WDh group. Bilateral LN hyperechogenicity was present in 17 WDn patients, and this phenomenon was stable over time. In the WDh group the number of patients with LN bilateral hyperechogenicity together with the mean area of LN increased, with significant change at 18 months (at 24 months the difference was not significant (Fig 2). Thalamic hyperechogenicity remained the least common finding: present in three patients with WDn and one patient with WDh. TCS follow-up results for each analysed time point are presented in the supplementary material (Fig. 2).
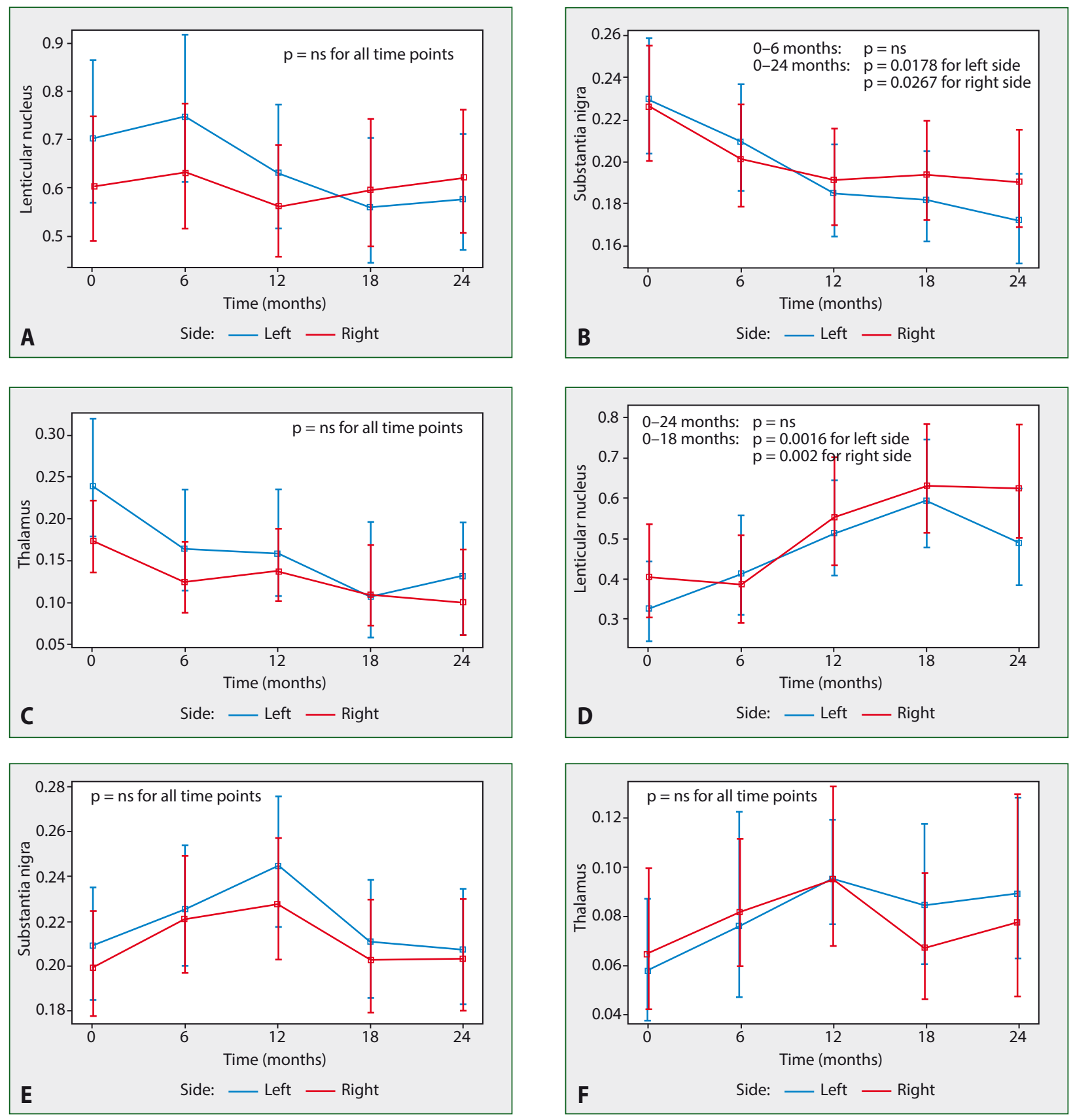

Figure 2. Transcranial sonography echogenicity changes in WDn and WDh patients in lenticular nucleus ( $A$ and $D$ respectively), in substantia nigra ( $\mathrm{B}$ and $\mathrm{E}$ respectively) and in thalamus ( $\mathrm{C}$ and $\mathrm{F}$ respectively) during 24-month follow-up. Mean \pm standard deviation; ns - not significant; WDn - Wilson's Disease patients with neurological manifestations; WDh - Wilson's Disease patients with hepatic manifestations 

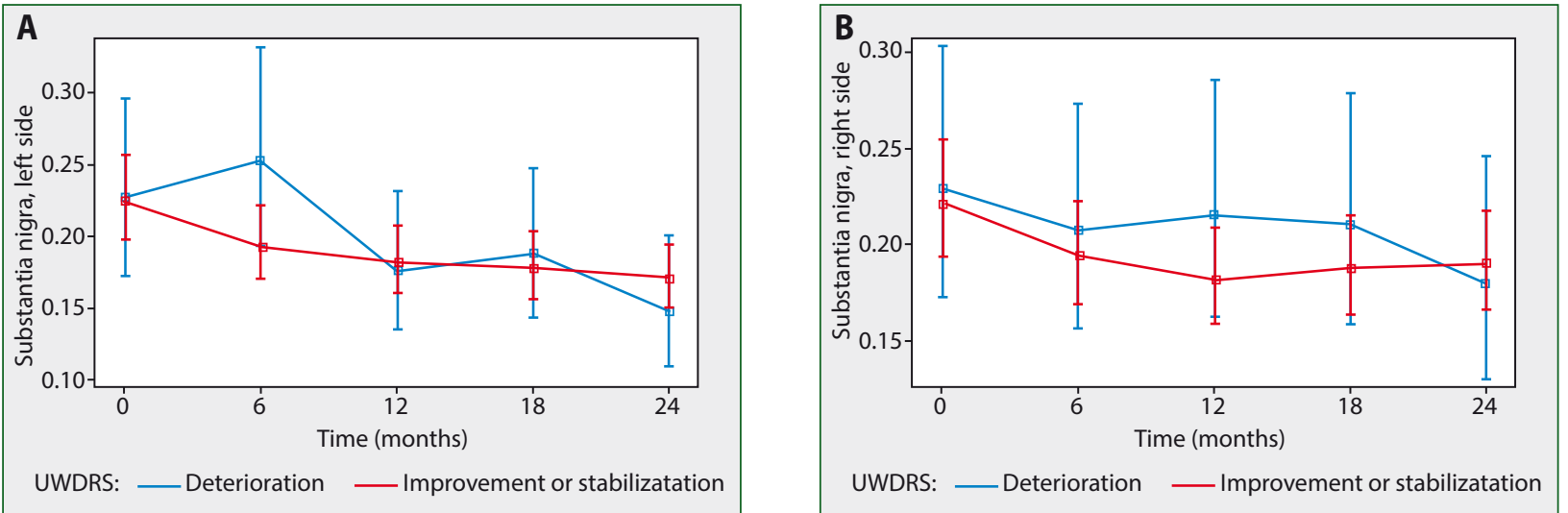

Figure 3. Transcranial sonography results from substantia nigra (A - left; B - right) of patients with neurological worsening or improvement/ stabilisation at month 24

No correlation between copper metabolism in patients with WDn and WDh and TCS results was found at any time point during the observation period. There was no correlation between UWDRS score and TCS results at any time point of observation. There were no differences in TCS results between deteriorated patients and patients with stable or improved outcomes in the WDn or WDh groups. SN echogenicity decreased over the study period in WDn, but this phenomenon was observed both in patients with improvements and deteriorations (Fig. 3).

\section{Discussion}

TCS is a valuable technique for the evaluation and diagnosis of various neurodegenerative disorders. It has previously been used for detecting changes in WD patients, [12-14], but never for monitoring de-coppering therapy. As MRI improvement after therapy introduction is observed in most cases $[2,9,18]$, we also expected that TCS results would improve. At baseline, the most common TCS finding in WD patients was LN hyperechogenicity. Bilateral hyperechogenicity was present in nearly all patients with WDn and in half of the patients with WDh. The mean area of LN echogenicity was higher for WDn. This is consistent with other TCS studies, where LN hyperechogenicity was found in nearly all patients [12] or $81 \%$ of WDn patients [13]. De-coppering therapy did not change these results: after two years of treatment, the number of WDn patients with LN hyperechogenicity was nearly the same, and actually increased in WDh. In our study, the area of LN was higher than has been previously reported, and did not decrease during the study period.

SN hyperechogenicity was less common in WD patients than LN echogenicity. We found SN hyperechogenicity in 50\% of WDn patients and 20\% of WDh patients at study entry.

SN hyperechogenicity was found more often in the present study than in previous investigations: one study reported $42 \%$ in WDn patients and 7\% in WDh patients [13], while another study observed marked SN hyperechogenicity in 25\% of WDn patients [12]. Both these previous studies were conducted in treated patients, and one of the most important findings of our study is that $\mathrm{SN}$ hyperechogenicity changed over time from treatment naivety. The number of patients with SN hyperechogenicity decreased and after two years of treatment SN hyperechogenicity was found only in one patient in the WDn group and four patients in the WDh group. Correlation of SN hyperechogenicity with clinical status was observed in one study [14], but not in two other studies [12, 13].

SN hyperechogenicity is observed in Parkinson's Disease (PD) and TCS is employed as an additional tool for PD diagnosis. SN hyperechogenicity is detectable in about $90 \%$ of patients even in the very early clinical stages of PD [19]. Contrary to our observation in WD patients, in PD patients SN hyperechogenicity is constant, probably due to neurodegenerative processes [19].

In the current study, thalamic hyperechogenicity was found in about $25 \%$ of WDn patients but in only one WDh patient. There have been inconclusive results from other studies, which have found thalamic hyperechogenicity in $50 \%$ of WD patients $[12,14$ ] or in no patients [13]. We found that thalamic hyperechogenicity also changed over the time; only three patients from the WDn group (15\%) had thalamic hyperechogenicity after 24 months.

Changes in TCS results observed after the introduction of de-coppering therapy raise questions regarding the nature of hyperechogenicity of deep brain nuclei. The exact answer is still unknown. TCS studies appear to show a relationship between echogenicity and metal accumulation. TCS hyperechogenicity was described in a group of PD patients as changes in SN that were thought to be due to iron accumulation [20], but other trace metals may also play an important role. In manganese-induced parkinsonism [21,22], patients have shown changes in $\mathrm{LN}$, but not in $\mathrm{SN}$ echogenicity, probably due to manganese 
accumulation. As MRI results improved, LN echogenicity also decreased, suggesting a correlation with the level of manganese in the brain [22]. LN hyperechogenicity is also found in more than $75 \%$ of patients with primary cervical and upper-limb dystonia, and in $31 \%$ of those with facial dystonia $[23,24]$. Following postmortem examination, significantly increased copper and manganese levels have been found in the globus pallidus and putamen of patients with primary dystonia compared to control brain samples [25]. Finally, postmortem TCS studies of WD patients have shown correlation of putaminal copper concentration and LN area [26]. At baseline, we found a correlation between $\mathrm{SN}$ hyperechogenicity and 24 -h copper urinary excretion. This also suggests a role for copper in SN hyperechogenicity.

It is also possible that hyperechogenicity is influenced by not only metal ions themselves, but also different ion binding partners such as neuromelanin and ferritin content, which might differ among patients [27]. Experimental studies in an animal model of $\mathrm{PD}$ show that $\mathrm{SN}$ hyperechogenicity is caused by structural changes, such as gliosis, rather than by increased iron concentration [28].

Our study has some limitations: first, a relatively small number of patients were included, which may limit conclusions regarding correlations. Secondly, more WDn patients were treated with DPA compared to WDh patients. However, we observed that TSC changed over time in both groups, so we believe that changes were caused by the therapy itself and not by the specific agent.

In conclusion, the most common change in TCS in WD patients was LN hyperechogenicity, which was observed despite treatment introduction and a two-year treatment observation period. SN hyperechogenicity was less common and decreased after treatment introduction, but there was no difference in the SN echogenicity decrease in patients who deteriorated compared to patients who improved or remained stable.

TCS hyperechogenicity in WD is probably due to copper accumulation, with the changes observed occurring as a result of de-coppering therapy introduction. TCS is not a valuable tool for predicting neurological deterioration in $\mathrm{WD}$ patients.

Acknowledgements: This study was supported by the Polish Ministry of Science and Higher Education grant No. NN402-472340

\section{Conflicts of interest: None declared}

\section{References}

1. Ala A, Walker AP, Ashkan K, et al. Wilson's disease. Lancet. 2007; 369(9559): 397-408, doi: 10.1016/S0140-6736(07)60196-2, indexed in Pubmed: 17276780.

2. Członkowska $A$, Litwin $T$, Dusek $P$, et al. Wilson disease. Nature Reviews Disease Primers. 2018; 4(1), doi: 10.1038/s41572-0180018-3.
3. European Association for Study of Liver. EASL Clinical Practice Guidelines: Wilson's disease. J Hepatol. 2012; 56(3): 671-685, doi: 10.1016/j.jhep.2011.11.007, indexed in Pubmed: 22340672.

4. Członkowska A, Litwin T, Karliński M, et al. D-penicillamine versus zinc sulfate as first-line therapy for Wilson's disease. Eur J Neurol. 2014; 21(4): 599-606, doi: 10.1111/ene.12348, indexed in Pubmed: 244447648.

5. Litwin T, Dzieżyc K, Karliński M, et al. Early neurological worsening in patients with Wilson's disease. Journal of the Neurological Sciences. 2015; 355(1-2): 162-167, doi: 10.1016/j.jns.2015.06.010.

6. King $A D$, Walshe JM, Kendall BE, et al. Cranial MR imaging in Wilson's disease. AJR Am J Roentgenol. 1996; 167(6): 1579-1584, doi: 10.2214/ajr.167.6.8956601, indexed in Pubmed: 8956601.

7. Kozić DB, Petrović I, Svetel M, et al. MR imaging of the brain in patients with hepatic form of Wilson's disease. Eur J Neurol. 2003; 10(5): 587-592, doi: 10.1046/i.1468-1331.2003.00661.x, indexed in Pubmed: 12940844.

8. Prashanth LK, Sinha S, Taly AB, et al. Do MRI features distinguish Wilson's disease from other early onset extrapyramidal disorders? An analysis of 100 cases. Mov Disord. 2010; 25(6): 672-678, doi: 10.1002/mds.22689, indexed in Pubmed: 20437536.

9. Sinha S, Taly AB, Prashanth LK, et al. Sequential MRI changes in Wilson's disease with de-coppering therapy: a study of 50 patients. Br J Radiol. 2007; 80(957): 744-749, doi: 10.1259/bjr/48911350, indexed in Pubmed: 17709362.

10. Kozić DB, Petrović I, Svetel M, et al. Reversible lesions in the brain parenchyma in Wilson's disease confirmed by magnetic resonance imaging: earlier administration of chelating therapy can reduce the damage to the brain. Neural Regen Res. 2014; 9(21): 1912-1916, doi: 10.4103/1673-5374.145360, indexed in Pubmed: 25558242.

11. Godau J, Berg D, Berg D, et al. Transcranial sonography in movement disorders. Lancet Neurol. 2008; 7(11): 1044-1055, doi: 10.1016/ S1474-4422(08)70239-4, indexed in Pubmed: 18940694.

12. Walter U, Krolikowski K, Tarnacka B, et al. Sonographic detection of basal ganglia lesions in asymptomatic and symptomatic Wilson disease. Neurology. 2005; 64(10): 1726-1732, doi: 10.1212/01. WNL.0000161847.46465.B9, indexed in Pubmed: 15911799.

13. Svetel M, Mijajlović M, Tomić A, et al. Transcranial sonography in Wilson's disease. Parkinsonism Relat Disord. 2012; 18(3): 234-238, doi: 10.1016/i.parkreldis.2011.10.007, indexed in Pubmed: 22024248.

14. Tribl GG, Trindade MC, Almeida KJ, et al. Quantitative transcranial sonography in Wilson's disease and healthy controls: Cut-off values and functional correlates. J Neurol Sci. 2018; 385: 69-74, doi: 10.1016/i. jns.2017.11.026, indexed in Pubmed: 29406916.

15. Gromadzka G, Schmidt HHJ, Genschel J, et al. p.H1069Q mutation in ATP7B and biochemical parameters of copper metabolism and clinical manifestation of Wilson's disease. Mov Disord. 2006; 21(2): 245-248, doi: 10.1002/mds.20671, indexed in Pubmed: 16211609.

16. Ferenci P, Caca K, Loudianos G, et al. Diagnosis and phenotypic classification of Wilson disease. Liver Int. 2003; 23(3): 139-142, doi: 10.1034/j.1600-0676.2003.00824.x, indexed in Pubmed: 12955875.

17. Członkowska A, Tarnacka B, Möller JC, et al. Unified Wilson's Disease Rating Scale - a proposal for the neurological scoring of Wilson's disease patients. Neurol Neurochir Pol. 2007; 41(1): 1-12, indexed in Pubmed: 17330175.

18. da Costa Md, Spitz M, Bacheschi LA, et al. Wilson's disease: two treatment modalities. Correlations to pretreatment and posttreatment 
brain MRI. Neuroradiology. 2009; 51(10): 627-633, doi: 10.1007/ s00234-009-0536-5, indexed in Pubmed: 19479249.

19. Gaenslen A, Unmuth B, Godau J, et al. The specificity and sensitivity of transcranial ultrasound in the differential diagnosis of Parkinson's disease: a prospective blinded study. The Lancet Neurology. 2008; 7(5): 417-424, doi: 10.1016/s1474-4422(08)70067-x.

20. Berg D, Roggendorf W, Schröder U, et al. Echogenicity of the substantia nigra: association with increased iron content and marker for susceptibility to nigrostriatal injury. Arch Neurol. 2002; 59(6): 999-1005, doi: 10.1001/archneur.59.6.999, indexed in Pubmed: 12056937.

21. Walter U, Dressler D, Lindemann C, et al. Transcranial sonography findings in welding-related Parkinsonism in comparison to Parkinson's disease. Mov Disord. 2008; 23(1): 141-145, doi: 10.1002/ mds.21795, indexed in Pubmed: 17987651.

22. Skowronska M, Dziezyc K, Członkowska A. Transcranial sonography in manganese-induced parkinsonism caused by drug abuse. Clin Neuroradiol. 2014; 24(4): 385-387, doi: 10.1007/s00062-013-0256-4, indexed in Pubmed: 24008860.

23. Naumann M, Becker G, Toyka KV, et al. Lenticular nucleus lesion in idiopathic dystonia detected by transcranial sonography. Neurology. 1996; 47(5): 1284-1290, doi: 10.1212/wnl.47.5.1284, indexed in Pubmed: 8909444.
24. Becker G, Naumann M, Scheubeck M, et al. Comparison of transcranial sonography, magnetic resonance imaging, and single photon emission computed tomography findings in idiopathic spasmodic torticollis. Mov Disord. 1997; 12(1): 79-88, doi: 10.1002/mds.870120114, indexed in Pubmed: 8990058.

25. Berg D, Weishaupt A, Francis MJ, et al. Increased tissue copper and manganese content in the lentiform nucleus in primary adult-onset dystonia. Ann Neurol. 1999; 46(2): 260-263, doi: 10.1002/1531-8249(199908)46:2<260::aid-ana18>3.0.c0;2-6, indexed in Pubmed: 10443894.

26. Walter U, Skowrońska M, Litwin T, et al. Lenticular nucleus hyperechogenicity in Wilson's disease reflects local copper, but not iron accumulation. J Neural Transm (Vienna). 2014; 121(10): 1273-1279, doi: 10.1007/s00702-014-1184-4, indexed in Pubmed: 24615184.

27. Zecca L, Berg D, Arzberger T, et al. In vivo detection of iron and neuromelanin by transcranial sonography: a new approach for early detection of substantia nigra damage. Mov Disord. 2005; 20(10): 12781285, doi: $10.1002 / \mathrm{mds} .20550$, indexed in Pubmed: 15986424.

28. Sadowski K, Szlachta K, Serafin-Król M, et al. Brain tissue echogenicity-implications for substantia nigra studies in parkinsonian patients. J Neural Transm (Vienna). 2012; 119(3): 363-367, doi: 10.1007/ s00702-011-0707-5, indexed in Pubmed: 21881837. 